Check for updates

Cite this: Chem. Commun., 2019, 55,3315

Received 8th January 2019,

Accepted 29th January 2019

DOI: $10.1039 / c 9 c c 00197 b$

rsc.li/chemcomm

\section{Liquid phase exfoliation of carbonate-intercalated layered double hydroxides $\dagger$}

\author{
Jose A. Carrasco, (D) $\ddagger^{a}$ Andrew Harvey $\ddagger^{b}$ Damien Hanlon, ${ }^{b}$ Vicent Lloret, ${ }^{c}$ \\ Dave McAteer, ${ }^{b}$ Roger Sanchis-Gual, (D) andreas Hirsch, (D) ${ }^{c}$ Frank Hauke, ${ }^{c}$ \\ Gonzalo Abellán, (D)*ac Jonathan N. Coleman (D)*b and Eugenio Coronado (D) ${ }^{a}$
}

Direct exfoliation of a carbonate layered double hydroxide (LDH) has been achieved by using a novel horn-probe sonic tip, avoiding the development of time-consuming anion-exchange reactions. The most suitable solvents were chosen based on the Hildebrand solubility parameters and the thickness of the exfoliated nanosheets confirmed unambiguously the successful delamination.

Layered double hydroxides (LDHs) are a class of ionic layered materials which display a brucite-like structure with the general formula $\left[\mathrm{M}^{\mathrm{II}}{ }_{1-\chi} \mathrm{M}^{\mathrm{III}}{ }_{\chi}(\mathrm{OH})_{2}\right]^{\chi+}\left(\mathrm{A}^{n-}\right)_{\chi / n} \cdot m \mathrm{H}_{2} \mathrm{O}{ }^{1,2}$ The main structure of an LDH highlights the cationic hydroxide sheets and the presence of the interlayer anions compensating the excess of positive charge (SI1, ESI†). In addition to that, one can find solvent molecules surrounding the structure. A key aspect of these materials is the ability to replace their interlayer anion thanks to anion exchange reactions. Hence, the rational choice of the metals and the interlayer anion can tune the final properties of the material, and LDHs are reported to exhibit a wide range of applications in diverse fields such as catalysis, anion exchange, sensing, magnetism and energy storage, among others. ${ }^{3-5}$ One of their most interesting features is their possibility to be exfoliated into 2D nanosheets, acting as macromolecular building blocks for the synthesis of more complex architectures while they retain the fundamental properties of the pristine bulk precursors such as the $2 \mathrm{D}$ magnetism or redox behaviour. ${ }^{5}$ In this context, the ionic nature of the LDHs hinders the exfoliation procedure in stark contrast with other $2 \mathrm{D}$ van der Waals materials, ${ }^{6}$ and time-consuming anion exchange reactions are mandatory to reduce the strength of the electrostatic interactions between the cationic layers and the interlayer anion. In a

\footnotetext{
${ }^{a}$ Instituto de Ciencia Molecular, Universitat de València, Catedrático José Beltrán 2, 46980, Paterna, Spain.E-mail: gonzalo.abellan@uv.es

${ }^{b}$ School of Physics and CRANN \& AMBER Research Centers, Trinity College Dublin, Dublin 2, Ireland. E-mail: colemaj@tcd.ie

${ }^{c}$ Department of Chemistry and Pharmacy, University Erlangen-Nürnberg, Henkestr. 42, 91054 Erlangen, Germany

$\dagger$ Electronic supplementary information (ESI) available. See DOI: 10.1039/ c9cc00197b

\$ JAC and $\mathrm{AH}$ contributed equally to this work.
}

typical synthesis, LDHs are obtained with carbonate $\left(\mathrm{CO}_{3}{ }^{2-}\right)$ in the interlayer space due to the higher affinity with respect to other anions, and therefore the direct exfoliation of a $\mathrm{CO}_{3}{ }^{2-}-\mathrm{LDH}$ is believed to be almost impossible. ${ }^{7}$ To overcome this inconvenience, other monovalent anions such as nitrate, chloride or anionic surfactants are introduced in the LDH structure by replacing the carbonate moiety and weakening the electrostatic interactions. ${ }^{8-10}$ In addition to that, formamide (which is highly toxic) stands out as the main solvent to carry out the exfoliation of LDHs, thanks to the ability to produce a swollen phase between the hydroxide sheets prior to the delamination. ${ }^{11}$ It is suggested that the driving force for the swelling of the LDH may be ascribed to the hydrogen bonding between the electronegative elements $(\mathrm{O}$ and $\mathrm{N})$ of the solvent and the hydroxide sheets, allowing its penetration into the gallery space and subsequent exfoliation. ${ }^{12}$ Nevertheless, the high boiling point of formamide $\left(210{ }^{\circ} \mathrm{C}\right)$ makes the evaporation difficult and therefore the processability of the nanosheets after the deposition on different substrates. Thus, alternative approaches are of utmost importance.

Here we report for the very first time the direct exfoliation of a $\mathrm{CO}_{3}{ }^{2-}-\mathrm{LDH}$ using liquid phase exfoliation via an ultrasonic tip. This method has reported successful results in the exfoliation of layered inorganic materials such as transition metal dichalcogenides, ${ }^{13}$ simple hydroxides ${ }^{14}$ black phosphorus ${ }^{15}$ or antimonene, ${ }^{16}$ to name a few. ${ }^{17,18}$ The use of tip sonication is novel in the LDH field, in stark contrast with the most typical bath sonication approach. ${ }^{10} \mathrm{~A}$ scheme describing the conventional and the novel tip sonication route for the LDHs can be found in Fig. 1A. The results were compared with the exfoliation of a dodecyl sulphate-LDH (DS-LDH), obtained after successive anion exchange reactions on the carbonate sample. Furthermore, different solvents were tested based on their Hildebrand solubility parameters to explore more suitable alternatives to formamide.

To start with, a highly crystalline $\mathrm{CoAl}-\mathrm{CO}_{3}$ sample was synthesized following the method described by Liu et al., ${ }^{7}$ (see SI2 and SI3, ESI $\uparrow$ for additional experimental details) using urea as an ammonia-releasing reagent to achieve a well-defined hexagonal morphology. ${ }^{3,19,20}$ The dodecyl-intercalated CoAl-DS sample was obtained after two successive anion exchange reactions, 


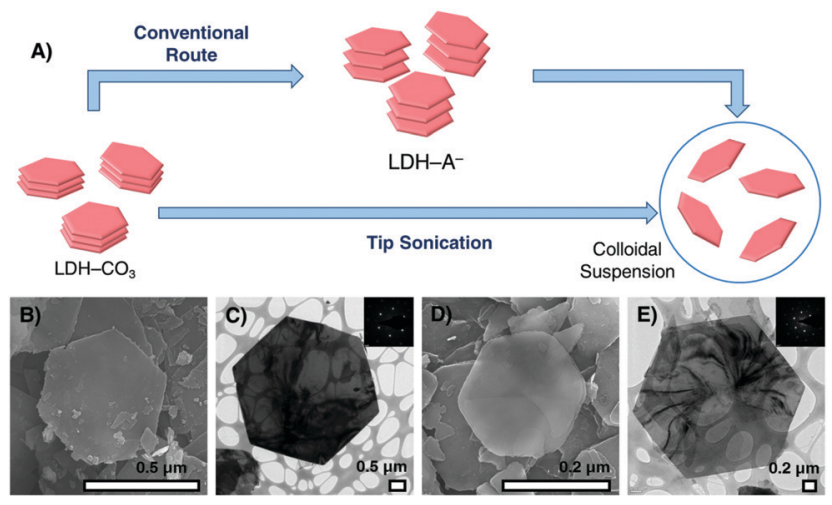

Fig. 1 (A) Scheme comparing the conventional exfoliation route and the tip sonication approach for LDHs. FESEM images of (B) $\mathrm{CoAl}-\mathrm{CO}_{3}$ and (D) COAl-DS. TEM images of (C) $\mathrm{COAl}-\mathrm{CO}_{3}$ and (E) COAl-DS highlighting in the inset the crystallinity of the material via SAED patterns.

first to nitrate using the acid-salt approach ${ }^{21}$ and afterwards to dodecyl sulphate. The synthesis of the LDH phase was confirmed by X-ray powder diffraction (XRPD) (SI4, ESI $\dagger$ ), exhibiting the main basal reflections for hydrotalcite-like materials (JCPDS 22-700). ${ }^{20,22}$ The main (003) peak is related to the basal space of the LDH material, therefore shifting towards lower $2-\theta$ values as the length of the intercalated anion increases. The basal spaces for the CoAl$\mathrm{CO}_{3}$ and CoAl-DS samples are found to be 7.6 and $26.7 \AA$, respectively. ${ }^{3}$ The nature of the interlayer species was also confirmed via attenuated total reflectance Fourier transform infrared (ATRFTIR) spectroscopy (SI4, ESI $\dagger$ ). The bands observed at $c a .3400$ and $1600 \mathrm{~cm}^{-1}$ are related to the $\mathrm{O}-\mathrm{H}$ bonding from the hydroxyl groups and the $\mathrm{H}_{2} \mathrm{O}$ molecules, respectively. The $\mathrm{CoAl}-\mathrm{CO}_{3}$ also exhibits a strong peak at $c a .1350 \mathrm{~cm}^{-1}$, indicative of the $\nu_{3}$ stretching mode of $\mathrm{CO}_{3}{ }^{2-}$ as the interlayer anion. ${ }^{20,23}$ Regarding the CoAl-DS, the most important peaks are the ones found at ca. 2917 and $2845 \mathrm{~cm}^{-1}$, related to the $\mathrm{C}-\mathrm{H}$ stretching. These bands are assigned to the presence of the surfactant and indicative of the successful intercalation of the dodecyl sulphate. ${ }^{3,24}$ According to the model proposed by Lagaly, we can expect a bilayer arrangement of the surfactant molecules between the hydroxide sheets with tilt angles between ca. 9 and $24^{\circ}{ }^{25}$ Thermogravimetric analysis (TGA) (SI4, ESI $\dagger$ ) also depicted the typical profile for the LDHs, with an initial weight loss in the $25-220{ }^{\circ} \mathrm{C}$ range, ascribed to the removal of adsorbed and interlayer $\mathrm{H}_{2} \mathrm{O}$ molecules (solvation molecules) and a second weight loss in the $220-600{ }^{\circ} \mathrm{C}$ range, related to the dehydroxylation of the layers and the decomposition of the interlayer anion. ${ }^{20,26}$ When larger molecules such as dodecyl sulphate are introduced in the interlayer space of the LDH, an increase in the final weight loss can be observed (from $70 \%$ to $40 \%$ after exchanging $\mathrm{CO}_{3}{ }^{2-}$ by $\mathrm{DS}^{-}$). ${ }^{3}$

The morphology of the bulk material was also imaged via electron microscopy (FESEM and TEM, Fig. 1B-E). Both samples exhibited a well-defined hexagonal morphology as expected for $-\mathrm{Al}^{3+}$ LDHs synthesized in the presence of ammonium releasing reagents (ARR) such as urea. ${ }^{7}$ This hexagonal morphology is conserved after the anion exchange reaction of $\mathrm{DS}^{-}$, and the average lateral size of CoAl- $\mathrm{CO}_{3}$ and CoAl-DS samples was found to be $c a .5 .0 \mu \mathrm{m}$ in both cases (see histograms in SI5, ESI $\dagger$ ). In addition to that, the selected area electron diffraction (SAED) pattern confirmed the high crystalline quality of the LDHs, in good agreement with that observed in XRPD. ${ }^{27}$ Additional FESEM images can be found in SI6, ESI. $\dagger$

The exfoliation of the materials was carried out following the methodology reported by some of us for other layered inorganic materials, consisting in the use of a tip sonication approach (see SI3, ESI $\dagger$ for additional experimental details). ${ }^{14-17}$ In nanomaterials, the stabilization is achieved when the solubility parameters between solvent and solute match. ${ }^{28}$ Due to this, not all solvents lead to stable dispersions. Therefore, the understanding of the solubility parameters developed by Hildebrand and Hansen becomes crucial to comprehend the chemistry that relies on the exfoliation into stable nanosheet dispersions. ${ }^{17,29}$ Herein, a complete solubility study on LDHs has been carried out using a total amount of 19 solvents based on their Hildebrand parameters. The complete solvent list can be found in SI7, ESI. $\dagger$ Both CoAl- $\mathrm{CO}_{3}$ and CoAl-DS were dispersed in each solvent as described in SI3, ESI ${ }^{\dagger}$ and the extinction spectra were measured using a UV-vis spectrometer. Extinction spectra become crucial for the nanomaterial dispersions since it takes into account the contribution from light scattering, non-trivial for the case of $2 \mathrm{D}$ nanosheets. ${ }^{30}$ Fig. $2 \mathrm{~A}$ and $\mathrm{B}$ depict the solubility diagrams for $\mathrm{CoAl}-\mathrm{CO}_{3}$ and CoAl-DS based on the value obtained at $450 \mathrm{~nm}$. Extinction spectra in the $350-500 \mathrm{~nm}$ region can be found in Fig. $2 \mathrm{C}$ and D. After the fitting of the results to a Gaussian function, ${ }^{15,31}$ as demonstrated with other materials exfoliated using this method, we found the best results for the $N$-cyclohexyl-2-pyrrolidone (CHP) and the 1-pentanol for the CoAl- $\mathrm{CO}_{3}$ and CoAl-DS samples, respectively, with Hildebrand parameters in the range of $c a$. 20.5-23 $\mathrm{M} \mathrm{Pa}^{0.5}$, similar to other layered materials. ${ }^{17}$ In addition, it is observed that some solvents
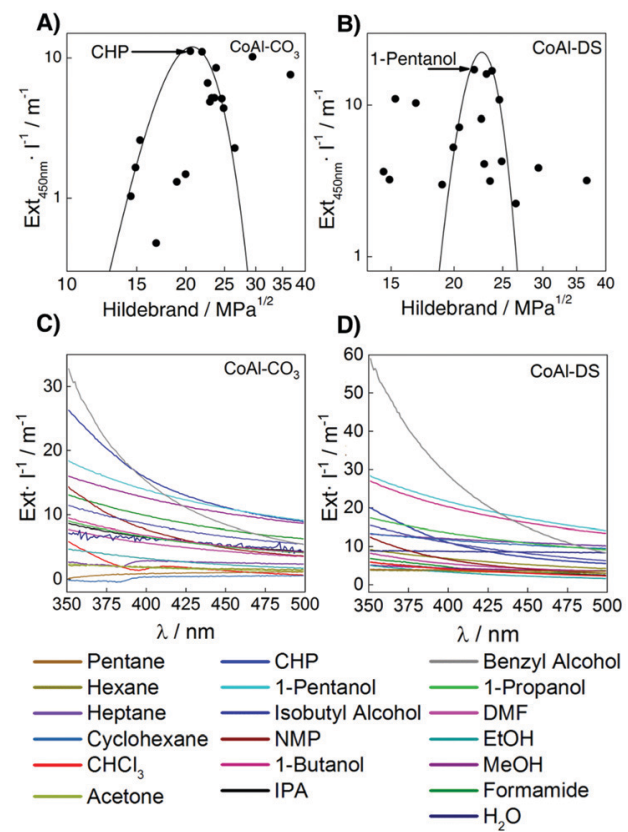

Fig. 2 Solubility diagrams of (A) $\mathrm{COAl}-\mathrm{CO}_{3}$ and (B) COAl-DS samples in a wide variety of solvents. Extinction spectra in the 350-500 nm region of (C) $\mathrm{CoAl}-\mathrm{CO}_{3}$ and (D) CoAl-DS. The solvent list table and the corresponding solubility parameters can be found in SI7, ESI. $\dagger$ 
exhibit higher concentration (ext/l) values than those observed for the typical formamide. For the $\mathrm{CoAl}-\mathrm{CO}_{3}$ sample, formamide displays a concentration (ext/l) value of $c a .7 .6 \mathrm{~m}^{-1}$, while solvents such as CHP, 1-pentanol, benzyl alcohol and $\mathrm{MeOH}$ surpass it with values of $11.2,11.1,8.5$ and $10.2 \mathrm{~m}^{-1}$, respectively. Regarding the CoAl-DS material, formamide depicts a concentration (ext/l) value of ca. $3.2 \mathrm{~m}^{-1}$, easily surpassed by most of the solvents employed (such as 1-pentanol, 1-butanol, benzyl alcohol or 1-propanol exhibiting values of 17.2, 16.1, 17.0 and $10.9 \mathrm{~m}^{-1}$, respectively). Nanosheet dispersions were characterized via TEM and atomic force microscopy (AFM). TEM images exhibited lateral sizes of several hundreds of nanometers for both samples, pointing out the fracture of the crystals after the sonication procedure (Fig. 3A and B). Additional TEM images can be found SI8 and SI9, ESI. $\dagger$ The stability of the $\mathrm{CoAl}-\mathrm{CO}_{3}$ suspension was analysed via UV-Vis, dynamic light scattering (DLS) and the Tyndall effect, exhibiting no reaggregation processes and an average size of $430 \mathrm{~nm}$ (SI10, ESI $\dagger$ ). On the other hand, the dispersions were spin coated on $\mathrm{SiO}_{2}$ substrates at 5000 r.p.m. and the thickness of the nanosheets was measured using AFM (Fig. 3C and D). Histograms for both samples are depicted in Fig. 3E and F, exhibiting an average thickness of ca. 11 and $10 \mathrm{~nm}$ for $\mathrm{CoAl}-\mathrm{CO}_{3}$ and CoAl-DS, respectively. Both results are statistically comparable and one order of magnitude lower than the thickness of the bulk materials (SI11, ESI $\dagger$ ), hence achieving for the first time the direct exfoliation of $\mathrm{a} \mathrm{CO}_{3}{ }^{2-}-\mathrm{LDH}$ by using any exfoliation technique. Since an ideal LDH monolayer is experimentally in the range of $0.6-0.8 \mathrm{~nm}$ in thickness, ${ }^{8}$ here we report the delamination into an average few-layer system. On top of that, thicknesses below $10 \mathrm{~nm}$ (especially in the low range 3-6 $\mathrm{nm}$ ) can be easily found according to the
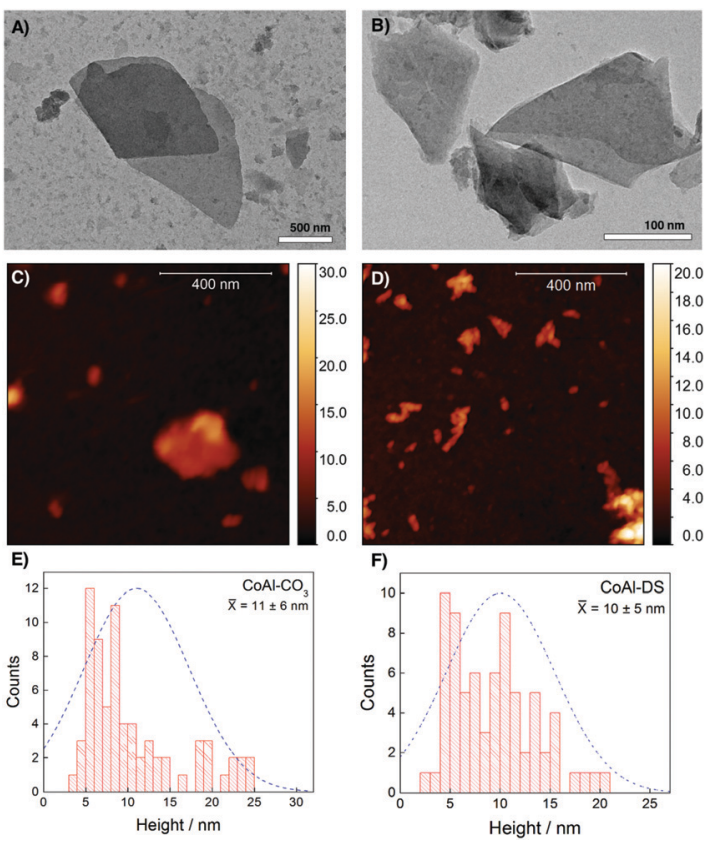

Fig. 3 TEM and AFM images of the ( $\mathrm{A}$ and $\mathrm{C}$ ) $\mathrm{COAl}-\mathrm{CO}_{3}$ and ( $\mathrm{B}$ and $\mathrm{D}$ ) CoAl-DS nanosheets in CHP and 1-pentanol, respectively. Histograms with the measured thickness of 75 nanosheets for (E) $\mathrm{COAl}_{-} \mathrm{CO}_{3}$ and (F) CoAl-DS deposited nanosheets. histogram, hence paving the way for the isolation of those nanosheets via size selection in cascade centrifugation. ${ }^{13}$ Height profiles for some individual nanosheets with a discussion on the lower limit of the thickness of nanosheets and the homogeneity after the exfoliation are depicted in SI12 and SI13, ESI. $\dagger$ An attempt to exfoliate $\mathrm{CoAl}-\mathrm{CO}_{3}$ using the conventional bath sonication approach led to unsuccessful exfoliation, as expected. This control experiment including the complete characterization can be found in SI14, ESI. $\dagger$

In addition, to demonstrate the usefulness of this approach we have explored the exfoliation of LDHs having different lateral dimensions and chemical composition. More concretely, we successfully exfoliated a nanometric $(c a .400 \mathrm{~nm}) \mathrm{CoAl}-\mathrm{CO}_{3}$ prepared using a traditional coprecipitation approach, ${ }^{26}$ and a hexagonal $\mathrm{NiFe}-\mathrm{CO}_{3}$ synthesized using the ARR route (SI15 and SI16, ESI $\dagger){ }^{8,20}$ In order to illustrate the potential of this new direct exfoliation route of LDHs via tip sonication, electrochemical measurements were tested on the $\mathrm{CoAl}-\mathrm{CO}_{3}$ exfoliated nanosheets (SI15 and SI16, ESI $\dagger$ ) and compared with the bulk materials as a catalyst for the oxygen evolution reaction (OER). ${ }^{32,33}$ Linear sweep voltammetry (LSV) was performed in order to evaluate the oxygen evolution activity in $1 \mathrm{M} \mathrm{NaOH}$ at $1 \mathrm{mV} \mathrm{s}^{-1}$ scan rate, obtaining the polarisation curves shown in Fig. 4A. An improvement in the OER performance is observed for the directly exfoliated $\mathrm{CoAl}-\mathrm{CO}_{3}$ nanosheets compared with the respective bulk LDHs, decreasing significantly the overpotential required for a current density of $10 \mathrm{~mA} \mathrm{~cm}{ }^{-2}$ to $c a .30 \mathrm{mV}$, and the Tafel slope to $c a .3 \mathrm{mV} \mathrm{dec}{ }^{-1}{ }^{10}$ These results are in good agreement with those reported for previously intercalated-LDHs and are indicative of the delamination of the material, as seen in Fig. 3E. ${ }^{10}$ It is important to notice the larger OER activity in nanosized LDHs, displaying lower overpotentials as well as Tafel slopes values (Fig. 4B and C). The enhancement in the OER is due to the increase in both the specific surface area and the number of active edge sites. ${ }^{10}$ Along this front, the Electrochemical Surface Areas (ECSAs) of the exfoliated and the bulk samples were analysed by obtaining the double layer capacitance (Fig. 4D). This value was calculated from cyclic voltammetry curves measured in a non-Faradaic region at different scan rates. The slopes of the fitted plots correspond to twice of the double layer capacitance. The exfoliated samples have a value $c a$. 1.4 higher than the bulk materials, confirming the successful exfoliation regardless of the size of the LDH used (see Fig. 4D). Last but not least, this tip exfoliation method was also tested on $\mathrm{NiFe}-\mathrm{CO}_{3} \mathrm{LDH}$ exhibiting higher improvement reducing the overpotentials and Tafel slopes values (SI15 and SI16, ESI $\dagger$ ).

In summary, we report for the first time the direct exfoliation of a $\mathrm{CO}_{3}{ }^{2-}-\mathrm{LDH}$ into few-layer nanosheets using a tip sonication methodology. The results were statistically equivalent with those observed for the delamination of a DS-LDH, with an average thickness of $c a .10 \mathrm{~nm}$ in both materials and the presence of a high number of nanosheets in the 3-6 nm range. A novel and complete solubility study on the $\mathrm{LDH}$ field based on the Hildebrand parameters was carried out for a wide variety of solvents, measuring their extinction spectra in an integrating sphere. The best solvents were found in the 20.5-23 $\mathrm{M} \mathrm{Pa}^{0.5}$ range, in 


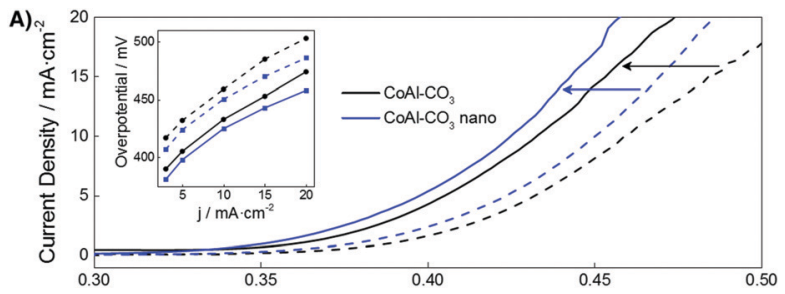

B)

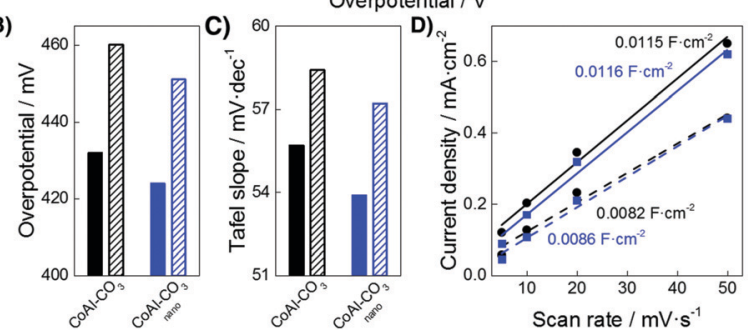

Fig. 4 Electrochemical performance of the $\mathrm{CoAl}-\mathrm{CO}_{3}$ samples (exfoliated LDHs in solid lines and bulk LDHs in dash lines). Inset: Overpotential measured at different current densities. (A) Linear sweep voltammetry curves performed at $1 \mathrm{mV} \mathrm{s}^{-1}$ in $1 \mathrm{M} \mathrm{NaOH}$. (B) Overpotentials required for $j=10 \mathrm{~mA} \mathrm{~cm}^{-2}$ and (C) Tafel slopes calculated from the LSV data. (D) Electrochemical surface area calculated from CVs performed in a nonFaradaic region at different scan rates.

good agreement with the expected values for other inorganic layered materials. These results open up a new approach in the field of the exfoliation of LDHs and pave the way for their processability.

This work was partially supported by the European Research Council (ERC Starting Grant 2D-PnictoChem 804110 and ERC Advanced Grant MOL-2D 788222). The research leading to these results was partially funded by the European Union Seventh Framework Programme (grant agreement no. 785219 and 604391, Graphene Flagship and 2D-INK project no. 664878). J. N. C. thanks the Science Foundation Ireland (SFI) funded centre AMBER (SFI/12/RC/2278). G. A. has received financial support through the Postdoctoral Junior Leader Fellowship Program from "la Caixa" Banking Foundation (LCF/BQ/PI18/11630018). G. A. thanks support by the Deutsche Forschungsgemeinschaft (DFG; FLAG-ERA AB694/ 2-1), and the Generalitat Valenciana (SEJI/2018/034 grant). A. H. thanks the SFB 953 "Synthetic Carbon Allotropes" funded by the DFG for support and the Cluster of Excellence "Engineering of Advanced Materials". R. S.-G. thanks the Ministerio de Ciencia, Innovación y Universidades for the F. P. U. fellowship. This work was supported by the Spanish MINECO (Grant MAT2017-89993-R and Excellence Unit María de Maetzu, MDM-2015-0538) and the Generalitat Valenciana (PROMETEO Program).

\section{Conflicts of interest}

There are no conflicts to declare.

\section{Notes and references}

1 C. Forano, T. Hibino, F. Leroux and C. Taviot-Guého, Developments in Clay Science, Elsevier, 2006, vol. 1, pp. 1021-1095.
2 G. Abellán, C. Martí-Gastaldo, A. Ribera and E. Coronado, Acc. Chem. Res., 2015, 48, 1601-1611.

3 J. A. Carrasco, S. Cardona-Serra, J. M. Clemente-Juan, A. Gaita-Ariño, G. Abellán and E. Coronado, Inorg. Chem., 2018, 57, 2013-2022.

4 M. Xu and M. Wei, Adv. Funct. Mater., 2018, 1802943.

5 Q. Wang and D. O'Hare, Chem. Rev., 2012, 112, 4124-4155.

6 K. S. Novoselov, A. Mishchenko, A. Carvalho and A. H. Castro Neto, Science, 2016, 353, aac9439.

7 Z. Liu, R. Ma, M. Osada, N. Iyi, Y. Ebina, K. Takada and T. Sasaki, J. Am. Chem. Soc., 2006, 128, 4872-4880.

8 G. Abellán, E. Coronado, C. Martí-Gastaldo, E. Pinilla-Cienfuegos and A. Ribera, J. Mater. Chem., 2010, 20, 7451-7455.

9 N. Mao, C. H. Zhou, D. S. Tong, W. H. Yu and C. X. Cynthia Lin, Appl. Clay Sci., 2017, 144, 60-78.

10 F. Song and X. Hu, Nat. Commun., 2014, 5, 4477.

11 R. Ma, Z. Liu, L. Li, N. Iyi and T. Sasaki, J. Mater. Chem., 2006, 16, 3809-3813.

12 T. Hibino, Chem. Mater., 2004, 16, 5482-5488.

13 C. Backes, B. M. Szydłowska, A. Harvey, S. Yuan, V. Vega-Mayoral, B. R. Davies, P. Zhao, D. Hanlon, E. J. G. Santos, M. I. Katsnelson, W. J. Blau, C. Gadermaier and J. N. Coleman, ACS Nano, 2016, 10, 1589-1601.

14 A. A. S. Rovetta, M. P. Browne, A. Harvey, I. J. Godwin, J. N. Coleman and M. E. G. Lyons, Nanotechnology, 2017, 28, 375401.

15 D. Hanlon, C. Backes, E. Doherty, C. S. Cucinotta, N. C. Berner, C. Boland, K. Lee, A. Harvey, P. Lynch, Z. Gholamvand, S. Zhang, K. Wang, G. Moynihan, A. Pokle, Q. M. Ramasse, N. McEvoy, W. J. Blau, J. Wang, G. Abellan, F. Hauke, A. Hirsch, S. Sanvito, D. D. O'Regan, G. S. Duesberg, V. Nicolosi and J. N. Coleman, Nat. Commun., 2015, 6, 8563.

16 C. Gibaja, D. Rodriguez-San-Miguel, P. Ares, J. Gómez-Herrero, M. Varela, R. Gillen, J. Maultzsch, F. Hauke, A. Hirsch, G. Abellán and F. Zamora, Angew. Chem., Int. Ed., 2016, 55, 14345-14349.

17 C. Backes, T. M. Higgins, A. Kelly, C. Boland, A. Harvey, D. Hanlon and J. N. Coleman, Chem. Mater., 2017, 29, 243-255.

18 V. Nicolosi, M. Chhowalla, M. G. Kanatzidis, M. S. Strano and J. N. Coleman, Science, 2013, 340, 1226419.

19 G. Abellán, J. L. Jordá, P. Atienzar, M. Varela, M. Jaafar, J. GómezHerrero, F. Zamora, A. Ribera, H. García and E. Coronado, Chem. Sci., 2015, 6, 1949-1958.

20 J. A. Carrasco, G. Abellán and E. Coronado, J. Mater. Chem. C, 2018, 6, 1187-1198.

21 N. Iyi, T. Matsumoto, Y. Kaneko and K. Kitamura, Chem. Mater., 2004, 16, 2926-2932.

22 L.-J. Zhou, X. Huang, H. Chen, P. Jin, G.-D. Li and X. Zou, Dalton Trans., 2015, 44, 11592-11600.

23 T. Xiao, Y. Tang, Z. Jia, D. Li, X. Hu, B. Li and L. Luo, Nanotechnology, 2009, 20, 475603.

24 R. B. Viana, A. B. F. da Silva and A. S. Pimentel, Adv. Phys. Chem., 2012, 2012, 1-14.

25 M. Meyn, K. Beneke and G. Lagaly, Inorg. Chem., 1993, 32, 1209-1215.

26 J. A. Carrasco, J. Romero, M. Varela, F. Hauke, G. Abellán, A. Hirsch and E. Coronado, Inorg. Chem. Front., 2016, 3, 478-487.

27 C. Hobbs, S. Jaskaniec, E. K. McCarthy, C. Downing, K. Opelt, K. Güth, A. Shmeliov, M. C. D. Mourad, K. Mandel and V. Nicolosi, npj 2D Mater. Appl., 2018, $2,4$.

28 P. May, U. Khan, J. M. Hughes and J. N. Coleman, J. Phys. Chem. C, 2012, 116, 11393-11400.

29 J. M. Hughes, D. Aherne and J. N. Coleman, J. Appl. Polym. Sci., 2013, 127, 4483-4491.

30 Light scattering reviews: single and multiple light scattering, ed. A. A. Kokhanovsky, Springer; Published in association with Praxis, Berlin; New York, Chichester, 2006.

31 A. Harvey, C. Backes, Z. Gholamvand, D. Hanlon, D. McAteer, H. C. Nerl, E. McGuire, A. Seral-Ascaso, Q. M. Ramasse, N. McEvoy, S. Winters, N. C. Berner, D. McCloskey, J. F. Donegan, G. S. Duesberg, V. Nicolosi and J. N. Coleman, Chem. Mater., 2015, 27, 3483-3493.

32 Y.-C. Liu, J. A. Koza and J. A. Switzer, Electrochim. Acta, 2014, 140, 359-365.

33 D. McAteer, I. J. Godwin, Z. Ling, A. Harvey, L. He, C. S. Boland, V. Vega-Mayoral, B. Szydłowska, A. A. Rovetta, C. Backes, J. B. Boland, X. Chen, M. E. G. Lyons and J. N. Coleman, Adv. Energy Mater., 2018, 8, 1702965. 